“C 2010 IEEE. Personal use of this material is permitted. Permission from IEEE must be obtained for all other uses, in any current or future media, including reprinting/republishing this material for advertising or promotional purposes, creating new collective works, for resale or redistribution to servers or lists, or reuse of any copyrighted component of this work in other works.” 


\title{
Performance Analysis of a Linear Synchronous Motor with HTS Bulk Magnets
}

\author{
Youguang Guo, Jianxun Jin, Jianguo Zhu, Haiyan Lu, Wei Xu
}

\begin{abstract}
The study on high temperature superconducting (HTS) bulk magnets and their application has attracted much attention. This paper presents the performance analysis of a linear synchronous motor (LSM) with HTS bulk magnets for driving an HTS maglev vehicle model. Finite element magnetic field analysis is carried out for computing the magnetic field distribution and key parameters of the LSM, and an equivalent electrical circuit is then applied to predict the motor performance, showing that the presented motor is appropriate for driving the model vehicle. The LSM has been prototyped, installed in the maglev vehicle model, and tested for validating the theoretical analysis.
\end{abstract}

Index Terms-- Finite element magnetic field analysis, linear synchronous motor, high temperature superconducting (HTS) bulk magnet, HTS maglev vehicle.

\section{INTRODUCTION}

$\mathrm{H}$ IGH temperature superconducting (HTS) materials and their applications have achieved significant development in the past two decades [1-2]. Different HTS materials have been developed in various forms, such as bulk, wire/tape and film, to suit various practical requirements. At the same time, extensive research has been conducted for a wide range of applications both in strong current aspects such as fault current limiters, transformers, energy storage devices, power cables, motors and generators, and HTS magnets; and in weak current aspects such as superconducting quantum interference devices (SQUIDs, e.g. magnetometers, gradiometers, microscopes, amplifiers), HTS microwave devices, and digital circuits and superconducting computers.

The HTS material can generate strong levitation force with very large force density and self-stabilizing feature and hence has attracted much research interest in maglev applications such as frictionless bearings [3] and transportation vehicles [4]. To investigate the HTS application in maglev transportation systems, the authors of this paper firstly developed a small-scale maglev train vehicle model with an overall size of $200 \times 50 \times 50 \mathrm{~mm}^{3}$ and a track of $2 \mathrm{~m}$ [5]. The vehicle is levitated by the interaction between HTS bulks placed on the ground and permanent magnets (PMs) mounted on the bottom of the vehicle. It has been proved that the system can produce inherent stability in

Y. G. Guo is with the School of Electrical, Mechanical and Mechatronic Systems, University of Technology Sydney, Sydney, NSW 2007, Australia (e-mail: Youguang.Guo-1@uts.edu.au).

J. X. Jin is with the Center of Applied Superconductivity and Electrical Engineering, University of Electronic Science and Technology of China, Chengdu, Sichuan 610054, China (e-mail: jxjin@uestc.edu.cn)

J. G. Zhu is with the School of Electrical, Mechanical and Mechatronic Systems, University of Technology Sydney, Sydney, NSW 2007, Australia (e-mail: joe@eng.uts.edu.au).

H. Y. Lu is with the School of Software, University of Technology Sydney, Sydney, NSW 2007, Australia (e-mail: helenlu@it.uts.edu.au).

W. Xu is with the School of Electrical, Mechanical and Mechatronic Systems, University of Technology Sydney, Sydney, NSW 2007, Australia (e-mail: wxu@eng.uts.edu.au). both vertical and lateral directions without any active control. The maglev vehicle model could be developed for train transportation or airplane launcher.

To drive the above-mentioned maglev vehicle model, a linear motor is naturally the best choice which provides many advantages over its rotary counterpart, such as high reliability, high efficiency, and low vibration and noise, thanks to the lack of mechanical transformation from rotary to linear movement. A linear permanent magnet synchronous drive system has been designed, manufactured and integrated into the system [5-6]. Experiments were carried out and the design concepts were validated. Furthermore, a linear induction motor drive system has also been proposed [7].

For more practical study, the second version of the vehicle model with $10 \mathrm{~m}$ long track has been developed. As shown in Fig. 1, the maglev system is used on both sides and each consists of HTS bulks on the vehicle and PM guide way on the ground. The linear drive system is placed in the middle, with a long primary and active track on the ground. The secondary of the linear motor is placed on the bottom of the vehicle, which can be a PM array for a linear synchronous motor [8] or a copper/aluminum sheet for a linear induction motor [9].

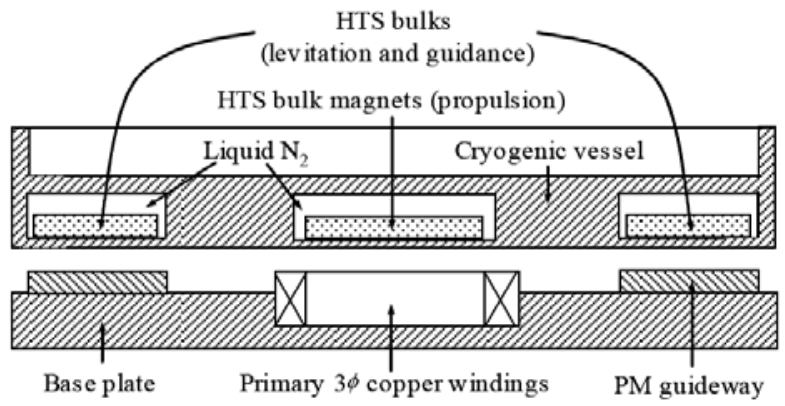

Fig. 1. Schematic view of a HTS maglev vehicle model with HTS bulk magnet LSM

The PMs in the secondary can be replaced by an array of YBCO HTS bulk magnets [10]. With the advance of HTS techniques, it has been reported that HTS bulks can trap a magnetic field well above $2.3 \mathrm{~T}$ at $77 \mathrm{~K}$ [11]. The application of such HTS bulk magnets for developing compact and high performance motors and actuators has attracted strong research interest recently [12].

This paper carries out further analysis of the presented HTS bulk magnet linear synchronous motor (LSM) for driving a HTS maglev vehicle model [10]. The numerical model of HTS bulk magnet is firstly investigated. Magnetic field finite element analysis (FEA) is then carried out for the whole motor region to accurately determine the magnetic field distribution and key parameters. The drive performance is predicted by using an equivalent electrical circuit. Some experimental results have been obtained on the motor prototype, validating the design concept and theoretical 
analysis.

\section{NUMERICAL MODEL OF HTS BULK MAGNET}

The HTS bulk can trap a magnetic field by zero-fieldcooled or field-cooled magnetization with steady magnetic field or pulse magnetic field. The flux density distribution of the bulk magnet can be calculated by the sandpile model in combination with the Biot-Savart law [13]. As illustrated in Fig. 2, the sandpile model divides the block magnet into $N_{L}$ layers and $N_{C}$ current loops. The loop current is given by

$$
I=J_{c} \Delta h \Delta w
$$

where $J_{c}$ is the critical current density of the bulk, $\Delta h$ the height of each layer, and $\Delta w$ the width of each current loop.

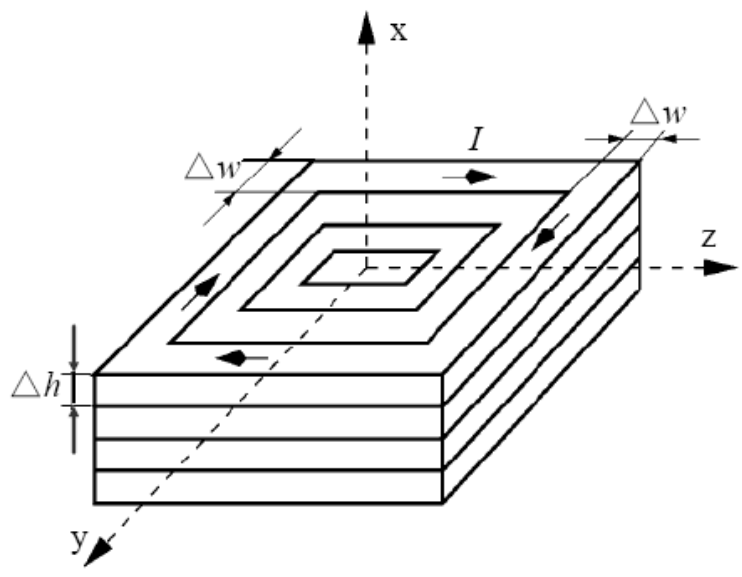

Fig. 2. Sandpile model of a block HTS bulk magnet

When $\Delta h$ and $\Delta w$ are sufficiently small, the loop current can be considered as a line current and the trapped field in the bulk magnet is given by the Biot-Savart law as

$$
d \mathbf{B}=\frac{\mu_{0} I}{4 \pi} \frac{1}{\|\mathbf{r}\|^{3}} d \mathbf{s} \times \mathbf{r}
$$

where $\mu_{o}$ is the permeability of vacuum, $\mathbf{r}$ the position vector from the current element $d \mathbf{s}$ to the observation point, and $\|\mathbf{r}\|$ is the length of $\mathbf{r}$.

\section{PARAMETER COMPUTATION AND PERFORMANCE ANALYSIS}

After zero-field-cooled or field-cooled magnetization, the HTS bulk magnets are installed on the vehicle bottom as the secondary of the linear motor. As shown in Fig. 3, six magnets are mounted along the vehicle movement direction with alternating poles of $\mathrm{N}$ and $\mathrm{S}$. In the transverse direction, three magnets with the same pole of $\mathrm{N}$ or $\mathrm{S}$ are used so that the total magnet width equals that of the stator core. The major dimensions of the LSM are listed in Table 1.

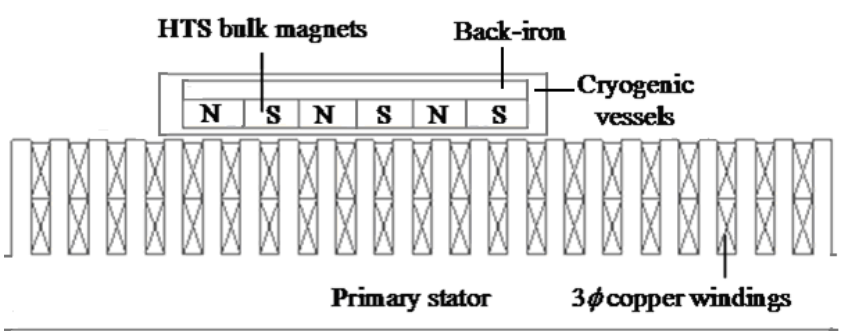

Fig. 3. Side view of the linear synchronous motor with HTS bulk magnets

TABLE I

MAJOR DIMENSIONS OF HTS BULK MAGNET LSM

\begin{tabular}{l|c}
\hline Stator tooth length (along movement direction) $(\mathrm{mm})$ & 10 \\
\hline Tooth width (transverse direction) $(\mathrm{mm})$ & 150 \\
\hline Slot depth (mm) & 100 \\
\hline Slot length (along movement direction) $(\mathrm{mm})$ & 20 \\
\hline Number of turns of copper coil & 200 \\
\hline Diameter of copper wire (mm) & 1.18 \\
\hline Pole pitch (mm) & 45 \\
\hline Main air gap length (vertical direction) $(\mathrm{mm})$ & 6 \\
\hline HTS bulk magnet length (along movement direction) $(\mathrm{mm})$ & 45 \\
\hline Total magnet width (transverse direction) $(\mathrm{mm})$ & 150 \\
\hline Magnet height (mm) & 12 \\
\hline Trapped magnetic field (T) & 0.6 \\
\hline Relative permeability of HTS bulk magnet & 0.4 \\
\hline
\end{tabular}

For computing the magnetic field distribution of the motor 2D finite element method is applied, which might be sufficient for theoretical analysis of this motor. It should be noted that 3D finite element analysis can be easily realized for more accurate analysis at the cost of more computation time. Fig. 4 shows the 2-D FEA solution region where the materials are numbered as (1) air, (2) HTS bulk magnet North, (3) HTS bulk magnet South, (4) back-iron, (5) stator core, and (6) stator winding.

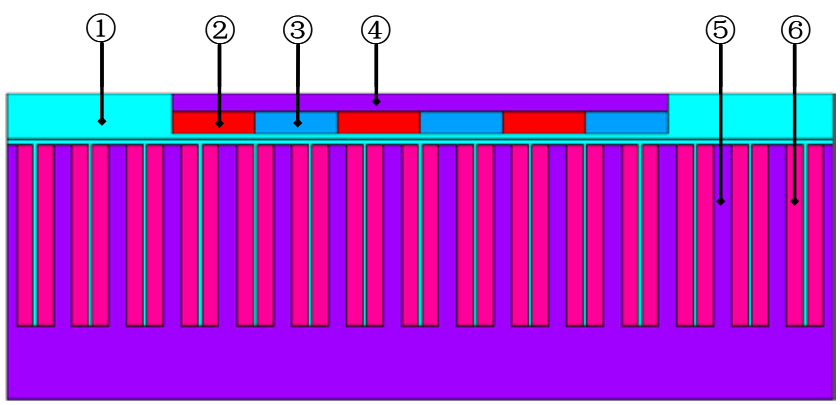

Fig. 4. 2-D FEA solution region of the HTS bulk magnet LSM

Based on the magnetic field FEA solutions, which have taken into account the detailed motor structure and magnetic nonlinearity of core materials, a number of key motor parameters can be accurately determined. For example, the PM flux, defined as the flux of one stator phase winding produced by the HTS bulk magnets, can be calculated from the no-load field distribution. Fig. 5 plots the waveform of PM flux against time or mover position, based on a series of FEA solutions at different mover positions in a pole-pair region. To reduce the computational error caused by the meshing, a separation mesh technique is used. The stator region and the mover region are separated along the middle line of the air gap and they are separately meshed. When the mover moves to a new position, e.g. a step of 15 electrical degrees, the stator and mover meshes are stitched together along the middle air gap line. To investigate the effect of the air gap length, the calculations with gaps of $0.8 \mathrm{~mm}, 2 \mathrm{~mm}$, and $4 \mathrm{~mm}$ are also given. It is seen that the flux magnitude increases with the decrease of the gap length; but this is constrained by the mechanical clearance, as well as the attractive force between stator and mover.

The PM flux waveform is an almost perfect sinusoid. When the vehicle moves, a back emf is induced in the stator 
winding and can be obtained by differentiating the PM flux against time. The motor back emf constant is computed as $5.036 \mathrm{Vs} / \mathrm{m}$, by

$$
K_{E}=\frac{\pi}{\tau} N_{s} \frac{\phi_{1}}{\sqrt{2}}
$$

where $\tau=45 \mathrm{~mm}$ is the pole pitch, $N_{s}=600$ is the number of effective turns of a phase winding, and $\phi_{1}=0.17 \mathrm{mWb}$ is the PM flux magnitude.

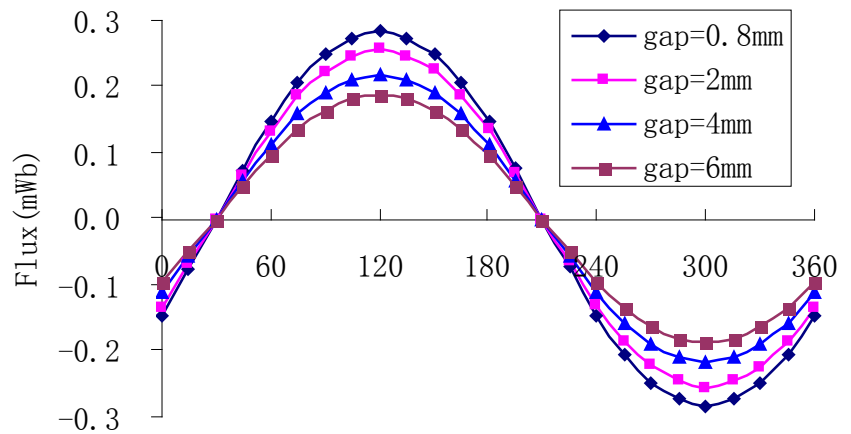

Mover position(ele. deg.)

Fig. 5. Per-turn PM flux of the stator phase winding

From the no-load field distribution, the cogging force can also be obtained by using the virtual work method or Maxwell stress tensor method. This force is unwanted, but its horizontal component (along the movement) may be used if a proper control is chosen. Fig. 6 shows the horizontal component of cogging torque against load angle. The scheme without back-iron is also given, showing that the employment of a back iron will increase the horizontal force. This is also applicable for the driving force produced by the magnetic interaction of bulk magnets and stator currents. Optimization design would be conducted for minimizing the cogging force.

The winding inductance can also be worked out from FEA field solutions. In this paper, the winding inductance is computed by using a modified incremental energy method [14]. Then the motor performance can be analyzed with an equivalent electric circuit as shown in Fig. 7, where $V_{1}$ is applied voltage, $E_{1}=K_{E} v$ is the back emf generated by the HTS bulk magnets on the mover, $v$ the vehicle speed, $v=2 \tau f, f$ the stator current frequency, $R_{1}$ the phase winding resistance, $X_{1}=2 \pi f L_{1}$ the synchronous reactance, and $L_{1}$ the synchronous inductance which equals the self inductance plus half the mutual inductance here.

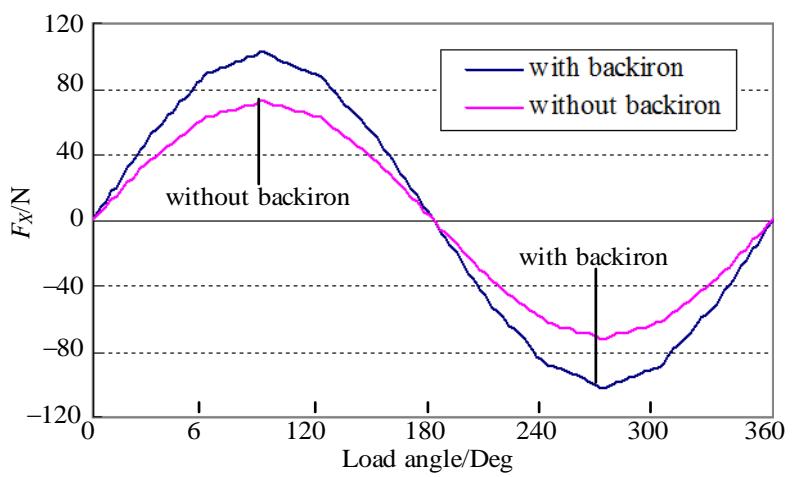

Fig. 6. Horizontal component cogging force against load angle

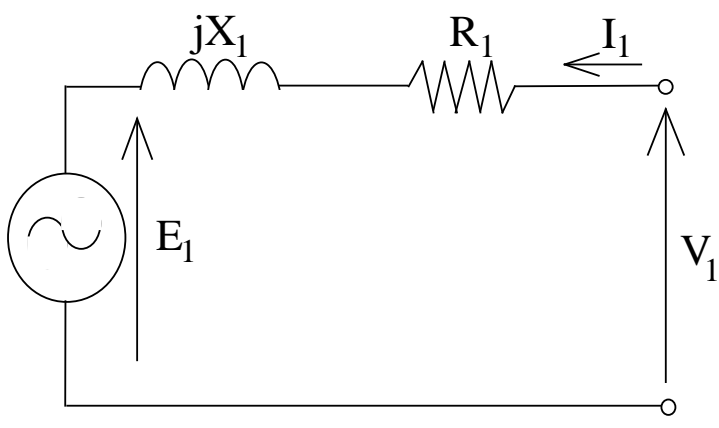

Fig. 7. Per-phase equivalent electrical circuit of PM synchronous motor

Under a control mode, the motor's mechanical characteristic, i.e. the relation of electromagnetic thrust force $F_{e m}$ against motor speed $v$, can be derived from the equivalent circuit. For example, the mechanical characteristic under the optimal brushless DC control is

$$
v=\frac{\sqrt{\left(\frac{R_{1} F_{e m}}{3}\right)^{2}+\left[\left(\frac{\pi L_{1} F_{e m}}{3 \tau K_{E}}\right)^{2}+K_{E}^{2}\right]\left[V_{1}^{2}-\left(\frac{R_{1} F_{e m}}{K_{T}}\right)^{2}\right]}-\frac{R_{1} F_{e m}}{3}}{\left(\frac{\pi L_{1} F_{e m}}{3 \tau K_{E}}\right)^{2}+K_{E}^{2}}
$$

Fig. 8 plots the computed thrust force against stator current. For comparison, the calculations with different trapped field of $0.35 \mathrm{~T}, 1 \mathrm{~T}$ and $2 \mathrm{~T}$ are also given.

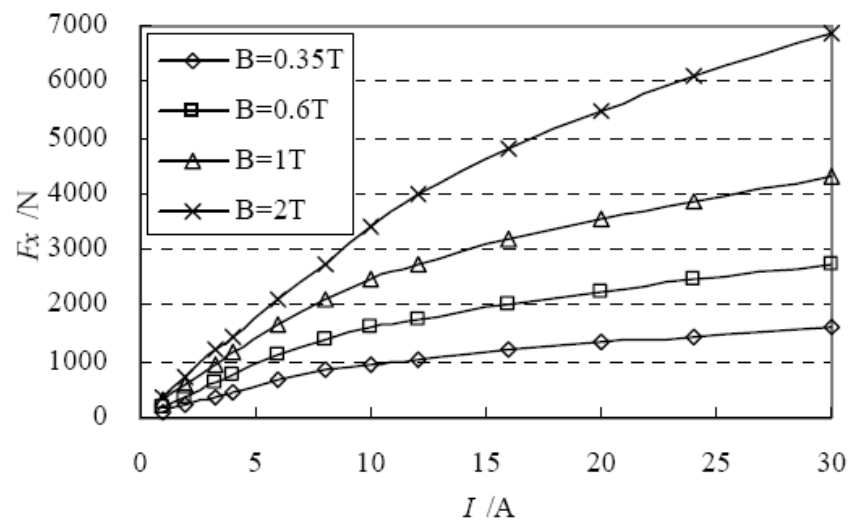

Fig. 8. Thrust force versus stator current with different trapped magnetic field

\section{PROTOTYPE EXPERIMENT}

Based on the above analysis, an HTS maglev vehicle with a HTS bulk magnet LSM drive has been developed. Fig. 9 illustrates the schematic view of the vehicle and Fig. 10 shows the photo of a practical prototype. For experimental convenience the mover is supported by two guide rails on both sides. The mover can slide freely along the guide rails, which will be replaced by the HTS maglev system in the final experiment. The LSM has been successfully operated with a voltage space vector PWM scheme. Fig. 11 shows the measured thrust force that the motor can produce at steady state at different speed or frequency. For comparison, the gap length between the stator and the mover is adjusted to $2 \mathrm{~mm}$ 
or $10 \mathrm{~mm}$ and the experimental results are also given.

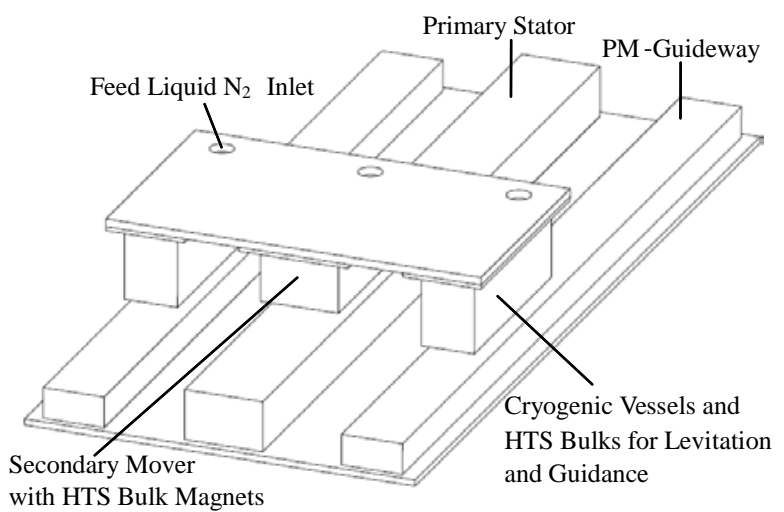

Fig. 9. Schematic view of the HTS maglev vehicle with HTS bulk magnet LSM drive

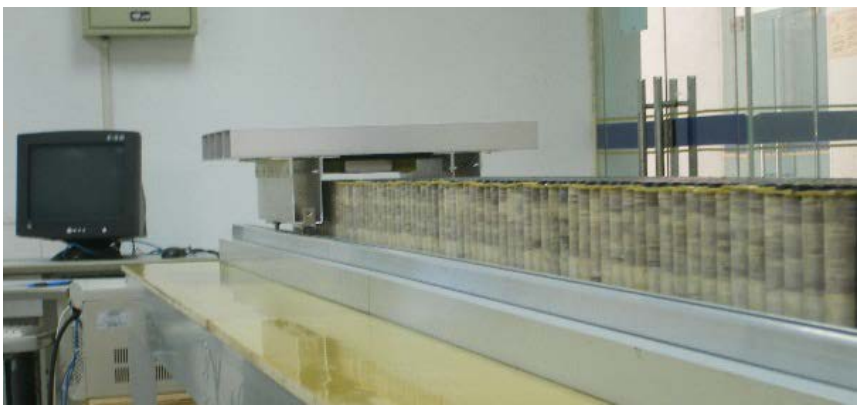

Fig. 10. Photo of experiment setup

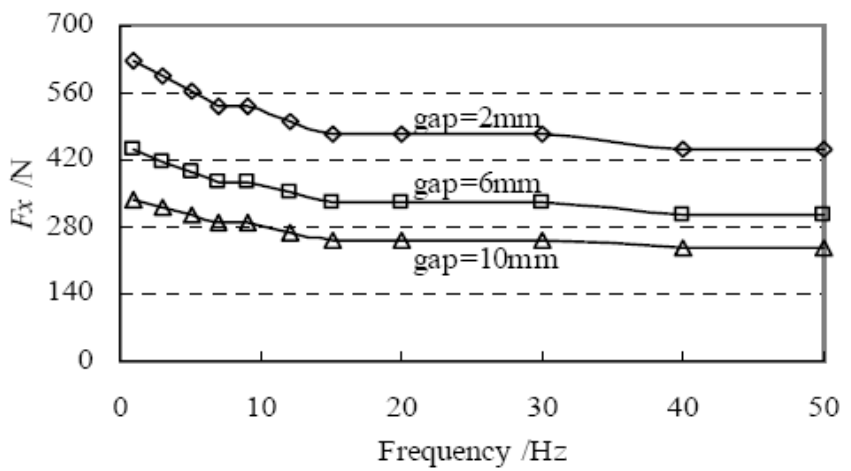

Fig. 11. Measured thrust force versus stator current frequency

\section{CONCLUSION}

This paper presents the performance analysis of a linear synchronous motor with HTS bulk magnets for driving a HTS maglev vehicle. The linear motor is composed of the primary stator on the ground and the secondary mover mounted on the vehicle bottom. The HTS bulk magnets are placed in a cryogenic vessel which is fixed on the secondary mover. Magnetic field finite element analysis is conducted to calculate the motor parameters and an equivalent electrical circuit is used to analyze the motor performance, e.g. the mechanical characteristic. An experimental prototype has been set up. Theoretical analysis and experimental results validate the design concept.

\section{REFERENCES}

[1] J. X. Jin, "High temperature superconductivity in the past twenty years part 1 - discovery, material and theory," HTS Special Issue of the Journal of Electronic Science and Technology of China, vol. 6, no. 2, pp. 225-236, June 2008.

[2] J. X. Jin, "High temperature superconductivity in the past twenty years part 2 - towards to practical applications," HTS Special Issue of the Journal of Electronic Science and Technology of China, vol. 6, no. 2, pp. 237-224, June 2008.

[3] T. Matsumura, S. Hanany, J. R. Hull, B. Johnson, and T. Jones, "Magnetic field in homogeneity and torque in high temperature superconducting magnetic bearings," IEEE Trans. on App. Super., vol. 15, no. 2, pp. 2316-2319, June 2005.

[4] H. W. Lee, K. C. Kim, and J. Lee, "Review of maglev train technologies," IEEE Trans. Magn., vol. 42, no. 7, pp. 1917-1925, July 2006.

[5] Y. G. Guo, J. X. Jin, J. G. Zhu, and H. Y. Lu, "Design and analysis of a prototype linear motor driving system for HTS maglev transportation," IEEE Trans. on App. Super., vol. 17, no. 2, pp. 20872090, June 2007.

[6] Y. G. Guo, J. X. Jin, L. H. Zheng, J. G. Zhu, and H. Y. Lu, "A permanent magnet linear synchronous motor drive for HTS maglev transportation system," HTS Special Issue of Journal of Electronic Science and Technology of China, vol. 6, no. 2, pp. 125-129, June 2008.

[7] Y. G. Guo, W. Xu, J. G. Zhu, H. Y. Lu, and J. X. Jin, "Design and analysis of a linear induction motor drive for a prototype HTS maglev transportation system," in Proc. IEEE Int. Conf. App. Super. \& Electromagnetic Devices, Chengdu, China, Sept. 2009, pp. 81-84.

[8] J. X. Jin and L. H. Zheng, "Performance analysis and optimization design of a HTS LSM," Journal of Science, Technology and Engineering, vol. 2, no. 1, pp. 19-22, 2008.

[9] L. H. Zheng, J. X. Jin, Y. G. Guo, and J. G. Zhu, "Design and characteristics analysis of long-primary single-sided linear induction motor," in Proc. IEEE Int. Conf. App. Super. \& Electromagnetic Devices, Chengdu, China, Sept. 2009, pp. 85-88.

[10] L. H. Zheng, J. X. Jin, Y. G. Guo, H. Y. Lu, and J. G. Zhu, "Design and Electromagnetic Analysis of a HTS Linear Synchronous Motor," in Proc. IEEE Int. Conf. App. Super. \& Electromagnetic Devices, Sept. 2009, Chengdu, China, pp. 5-10.

[11] N, Sakai, M. Kita, S. Nariki, et al., "Field trapping property of Gd-BaCu-O bulk superconductor $140 \mathrm{~mm}$ in diameter," Physica C, vol. 445448, pp. 339-342, 2006.

[12] A. Takahashi, H. Ueda, and A. Ishiyama, "Trial production and experiment of linear synchronous actuator with field-cooled HTS bulk secondary," IEEE Trans. on App. Super., vol. 13, no. 2, pp. 22512254, June 2003.

[13] H. Fukai, M. Tomita, M. Murakami, and Nagatomo, "Numerical simulation of trapped magnetic field for bulk superconductor," Physica C, vol. 357-360, no. 2 (SUPPL), pp. 774-776, 2001.

[14] J. X. Chen, Y. G. Guo, and J. G. Zhu, "Development of a high speed permanent magnet motor for driving embroider machines," IEEE Trans. Magn., vol. 43, no. 11, pp. 4004-4009, Nov. 2007.

\section{BIOGRAPHIES}

Youguang Guo (S’02-M’05-SM’06, IEEE) was born in Hubei, China in 1965. He received the B.E. degree from Huazhong University of Science and Technology (HUST), China in 1985, the M.E. degree from Zhejiang University, China in 1988, and the PhD degree from University of Technology, Sydney (UTS), Australia in 2004, all in electrical engineering. His research fields include measurement and modeling of magnetic properties of magnetic materials, numerical analysis of electromagnetic field, electrical machine design and optimization, power electronic drives and control. In these fields, he has published over 230 refereed technical papers including 117 journal articles.

Jianxun Jin, Professor, Ph.D., Ph.D. adviser, is currently the Director of the Center of Applied Superconductivity and Electrical Engineering at the University of Electronic Science and Technology of China. His research interests mainly include applied superconductivity, electromagnetic devices, electric machine, electric power, control, measurement and energy efficiency technology

Jianguo Zhu received his BE in 1982 from Jiangsu Institute of Technology, China, ME in 1987 from Shanghai University of Technology, China, and Ph.D in 1995 from University of Technology, Sydney (UTS), 
Australia, He currently holds the positions of Professor of Electrical Engineering and Head for School of Electrical, Mechanical and Mechatronic Systems at UTS, Australia. His research interests include electromagnetics, magnetic properties of materials, electrical machines and drives, power electronics, and renewable energy systems.

Haiyan Lu received the B.E. and M.E. degrees (Electrical Engineering) from Harbin Institute of Technology, China, in 1985 and 1988, respectively, and the $\mathrm{PhD}$ degree (Engineering) from University of Technology, Sydney (UTS), Australia, in 2002. She is currently with the School of Software, Faculty of Engineering and Information Technology, University of Technology, Sydney, Australia. Her research interests include modeling and numerical simulation of magnetic properties of materials, optimal design of electrical machines and drives, heuristic optimization techniques and intelligent web-based systems. She has published over 50 refereed journal articles and conference papers.

Wei Xu was born in Chongqing, China, in 1980. He received the B.E., B.A., and M.E. degrees from Tianjin University, Tianjin, China, in 2002 and 2005, respectively, and the Ph.D. degree from the Institute of Electrical Engineering, Chinese Academy of Sciences, Beijing, China, in 2008, all in electrical engineering. He is currently a Postdoctoral Fellow with the Center for Electrical Machines and Power Electronics, University of Technology Sydney (UTS), Sydney, Australia, where his research is supported by the Early Career Researcher Grant and partly supported by the Chancellor's Postdoctoral Research Fellowship, both at UTS. His research interests mainly include electromagnetic design and performance analysis of linear/rotary machines, including induction, permanent magnet, switched reluctance, and other emerging novel structure machines. 